\title{
Study on Noise Prediction and Reduction in Coupled Workshops Using SEA Method
}

\author{
Ye Lei, ${ }^{1,2}$ Jie Pan, ${ }^{1}$ and Meiping Sheng ${ }^{2}$ \\ ${ }^{1}$ School of Mechanical and Chemical Engineering, The University of Western Australia, Nedlands, Perth, WA 6009, Australia \\ ${ }^{2}$ School of Marine Engineering, Northwestern Polytechnical University, Xi'an 710072, China
}

Correspondence should be addressed to Ye Lei, leixiaoye@mail.nwpu.edu.cn

Received 31 January 2011; Accepted 28 March 2011

Academic Editor: M. Ichchou

Copyright () 2011 Ye Lei et al. This is an open access article distributed under the Creative Commons Attribution License, which permits unrestricted use, distribution, and reproduction in any medium, provided the original work is properly cited.

\begin{abstract}
A theoretical model for predicting noise reduction in coupled workshops is presented by using statistical energy analysis (SEA) method. An opening between the coupled workshops is considered into the theoretical model properly. The leakage issue is dealt with in the process of SEA modeling. An experiment is also carried out. A reasonable agreement between the prediction of noise reduction and the experimental data is observed. Moreover, it is concluded from the simulations that the sound energy transmit through the opening was the most important way to affect the noise reduction and the leakage is a significant element to influence the effect of noise treatment.
\end{abstract}

\section{Statement of the Problem}

The noise exposure standard of workplace in Australia is that the average exposure is $85 \mathrm{~dB}(\mathrm{~A})$ in the form of overall sound pressure level over an eight-hour working day [1]. This regulation does not mean that below $85 \mathrm{~dB}(\mathrm{~A})$ is safe, but that an eight-hour exposure of $85 \mathrm{~dB}(\mathrm{~A})$ is considered to represent an acceptable level of risk to human hearing health. It is stated that if the noise level exceeds the regulation, action must be taken immediately. The investigated object in this paper is large coupled workshops, having a pump in one of them used for providing motility for water circulation, a double configured plasterboard placed in the middle of the workshops, and an opening beyond the plasterboard. When the pump runs, people in the receiving room feel so noisy that they cannot work efficiently and comfortably. Therefore, acoustical responses in both workshops and noise reduction between them should be assessed. If the overall sound pressure level exceeds $85 \mathrm{~dB}(\mathrm{~A})$, noise treatment either on the noisy source, or on the propagation route should be carried out. In order to guide the noise treatment work, the effect of noise reduction after control also needs to be estimated. The configuration of the coupled workshops is shown in Figure 1. The left subfigure is the coupled workshops. The length of both room volumes is $15 \mathrm{~m}$. The right subfigure describes the detailed configuration in the middle of coupled workshops, including an opening and double plasterboards. The plasterboards are fixed by screw and metal stud frame. Some rayon material fills the air gap between the plasterboards.

\section{Literature Review}

In practical project, various requirements need to be considered in the process of constructing buildings, thereby, no architecture can be built perfectly. Under the desire of better habitation and working environment, buildings are required to be constructed as ideally as possible. Therefore, investigation on acoustical properties of buildings is a meaningful topic, and the interrelated research has been developed for many years.

Furukawa et al. [2] proposed a two-dimensional SEA model for analyzing the characteristics of structure-born sound in a slender building and obtained the sound attenuation per unity distance. Wentang and Attenborough [3] also predicted sound pressure levels in coupled rooms using SEA method and the results are closer to the measured results than those obtained by the statistical acoustical method. In their study, the coupling between the window, doors, and walls is ignored, because the walls are so thick that most of 


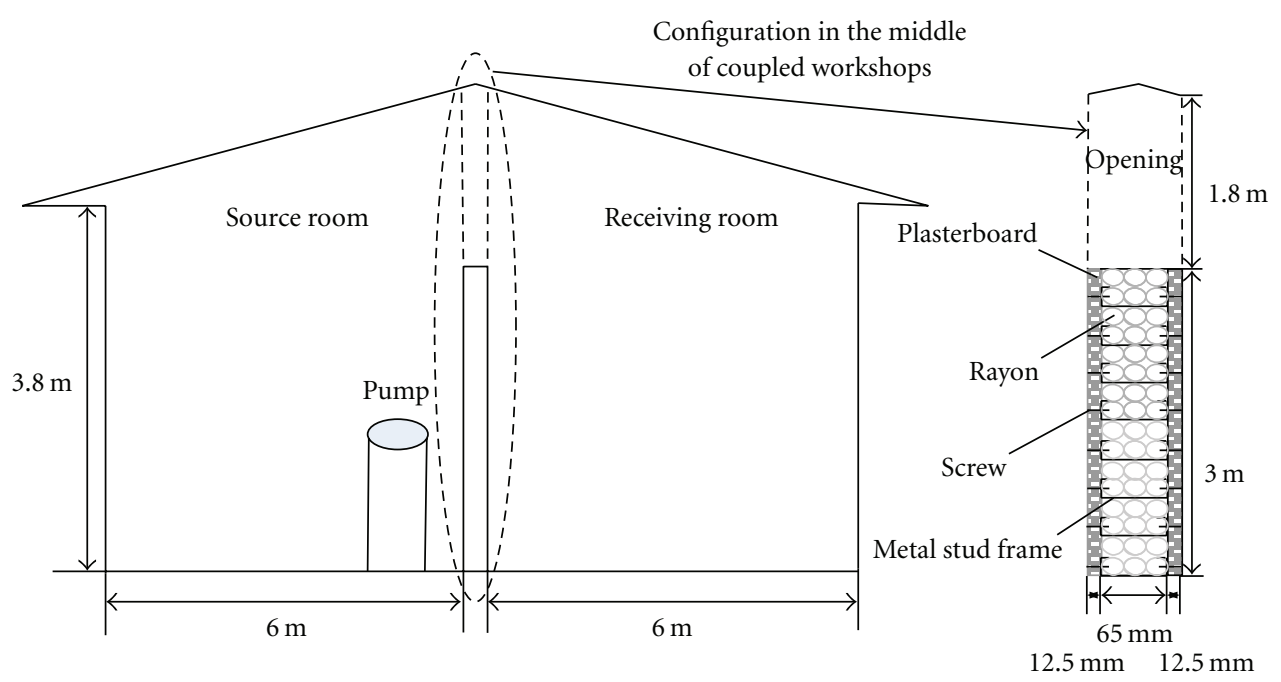

FIGURE 1: The configuration of the coupled workshops.

the energy is dissipated within them and little is reradiated to other subsystems. In reality, leakage often exists in walls and windows, and many scholars found that it is crucial to transfer sound energy. Early studies by Sauter and Soroka [4] developed a theoretical model for two reverberant rooms with a slot between them. The slot was treated as a rigid rectangular piston while using the impedance function to express the relationship of sound pressures between the room volumes and slot, and only plane wave propagation was assumed through the slot. More recently, Billon et al. [5] analyzed coupled rooms with an aperture in them and proposed a diffusion model used for predicting acoustical responses taking the location into account, and the calculated results agreed well with the experimental data and those gained by the ray method. Franck et al. [6] gave a detailed review of methods for modeling apertures and presented a rigorous model for predicting the transmission loss through the aperture. In the model of Franck et al., propagating and evanescent acoustic modes were considered inside the aperture, and modal radiation impedance matrix was coupled with these modes in terms of modal contribution factors.

The aims of this paper are to take an opening into SEA model properly, to analyze the effect of leakage based on the model suggested by Gomperts and Kihlman [7], and to give constructive guidance for noise control. Section 3 presents the theoretical SEA models before and after noise control; Section 4 discusses the experimental studies, including measuring the sound pressure levels and reverberation times; Section 5 addresses the comparison of the predicted and measured results and analyzes the effect of opening and leakage on energy transfer paths; Section 6 summarizes this research.

\section{Theoretical Analysis}

3.1. SEA Model. In the process of building SEA model, it is complicated and of no need to express the whole vibroacoustic system exactly because of the basic statistical principle.
For simplicity, it is assumed that there is no coupling between walls, floors, doors, windows, and ceilings. There are some equipments and furniture in the workshops. They provide necessary scattering conditions to satisfy statistical principle and are out of consideration in the SEA model.

Based on the above assumptions, the power flow relationships of subsystems are shown in Figure 2 , where $\Pi_{i \text {,in }}$ is the input power to subsystem $i, \Pi_{i \text {,diss }}$ is the dissipated power in subsystem $i$, and $\Pi_{i, j}$ is the power exchanged between subsystem $i$ and subsystem $j$.

A room system is divided into six kinds of sub-systems, including the room volume, the walls, the doors, the floor, the ceiling, and the windows. The power from the pump is inputted into the source room volume. The walls between the source room volume and the receiving room volume have direct coupling. The same coupling between ceilings exists. The door subsystem has coupling with the plasterboards. The path of transferring sound energy through the opening is represented as the direct coupling between the source room volume and the receiving room volume. Different from in the conventional SEA method, in the improved SEA model the indirect coupling between the room volumes and air gap inside the double plasterboards is ignored, but the nonresonant vibration and transmission of plasterboards [8] are included. The metal stud frame connecting two plasterboards is treated as beam class subsystems.

The basic equation for expressing the energy flow relationships between the $i$ th subsystem and other subsystems in SEA model is

$$
\omega \eta_{i} E_{i}-\omega \sum_{j \neq i}^{N} \eta_{j, i} E_{j}=\Pi_{i}
$$

where the subscripts $i$ and $j$, represent the subsystem $i$ and $j$ respectively, $\omega$ is the angle frequency, $\eta_{i}$ is the DLF, $\eta_{i, i}$ is the coupling loss factor (CLF), $E_{i}$ is the average modal energy, $\Pi_{i}$ is the input power to subsystem $i$ from outside environment, and $N$ is the total number of subsystems. If there is no 


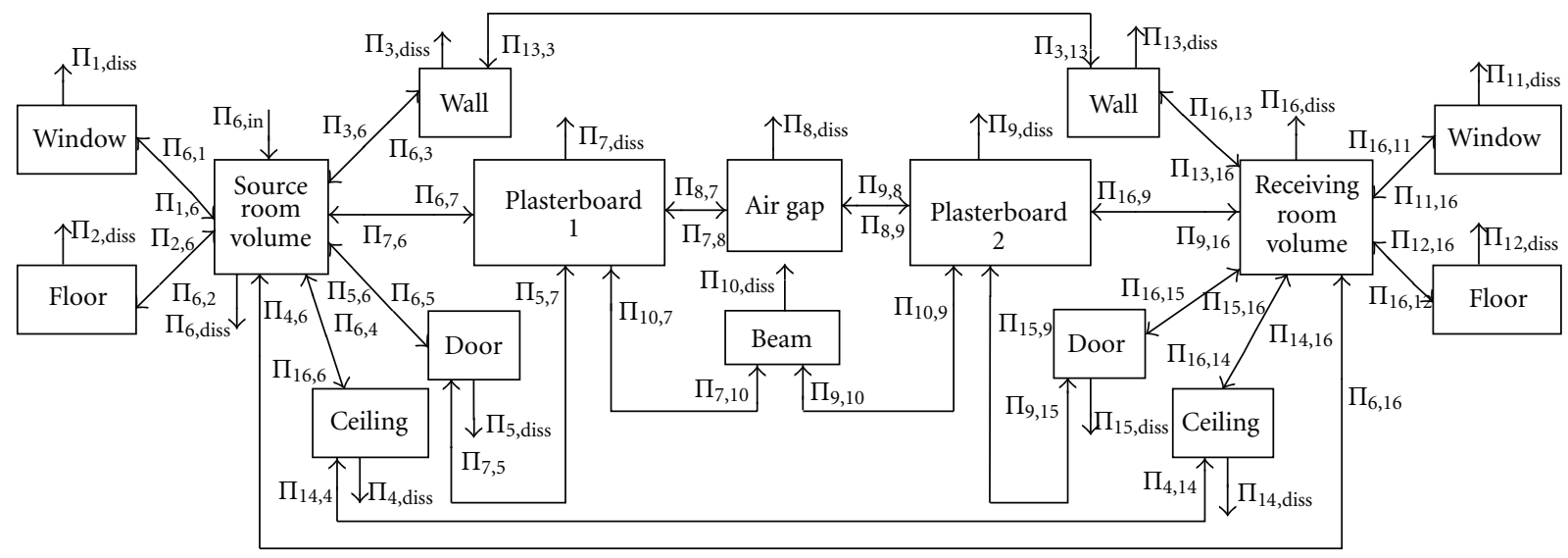

FIGURE 2: The energy flow relationships between subsystems before noise control.

coupling between two subsystems, the CLF in (1) will be set to zero. The input power equals zero when there is no direct power from outside excitation.

The structural-structural CLF is calculated by [9]

$$
\eta_{s i, s j}=\frac{L_{s i, s j} C_{g, s i}}{\pi \omega S_{s i}} \tau_{s i, s j},
$$

where $L_{s i, s j}$ is the junction length, $\tau_{s i, s j}$ is the power transmission coefficient through the junction connecting structural subsystem $i$ and $j$, and $C_{g, s i}$ and $S_{s i}$ are the group velocity and area of structural element $i$.

The structural-acoustical and acoustical-structural CLFs are computed by [10]

$$
\begin{aligned}
& \eta_{s i, a j}=\frac{\rho_{a j} c_{a j}}{\omega \rho_{s i}} \sigma_{s i}, \\
& \eta_{a j, s i}=\frac{\rho_{a j} c_{a j}^{2} S_{s i} f_{c, s i}}{8 \pi V_{a j} \rho_{s i} f^{3}} \sigma_{s i},
\end{aligned}
$$

where $\rho_{a j}, c_{a j}$, and $V_{a j}$ are the medium density, sound velocity, and volume of room space $j$, respectively, and $\rho_{s i}, f_{c, s i}$, and $\sigma_{s i}$ are the surface density, critical frequency, and radiation efficiency $[8,11]$ of substructure $i$.

The acoustical-acoustical CLF is simulated as follows [9]

$$
\eta_{a i, a j}=\frac{c_{a i} S_{p}}{4 \omega V_{a i}} \tau_{a i, a j}
$$

where $c_{a i}$ and $V_{a i}$ are the sound velocity and volume, respectively, $S_{p}$ is the area of structure used for separating room volumes, and $\tau_{a i, a j}$ is the transmission coefficient between the directly coupled room volumes. The transmission coefficient is supposed to be equal unity [6] for the case of opening and calculated by the model of Gomperts [7] for the case of leakage.

Finally, the average model energy of each subsystem can be obtained by solving (1). The squared sound pressure of acoustical subsystem is expressed by the average modal energy,

$$
p_{a i}^{2}=\frac{\rho_{a i} c_{a i}^{2}}{V_{a i}} E_{a i} .
$$

The details of subsystems and energy flow relationships after treatment are shown in Figure 3. Different from in Figure 2, in Figure 3 the direct acoustical-acoustical coupling between the two room volumes is ignored, in the assumption that the craftwork is so consummate that no leakage existed. The opening is filled with double plasterboards, whose material and configuration are the same as the existing one. The nominations are accordant with those in Figure 2.

3.2. Contribution Analysis. In order to guide the control work, investigation on energy transfer path is necessary. The contribution factor of the energy transfer path is defined as the ability of transferring energy and related with DLF and CLF of subsystems taking part in corresponding path. For an interesting subsystem, the total contribution from all the energy transfer paths is defined as a coefficient $D$, which is the average model energy ratio of the interesting subsystem and the source subsystem that is excited by external power directly

$$
D=\frac{E_{r}}{E_{s}}=\frac{E_{r}^{1}+E_{r}^{2}+\cdots+E_{r}^{i}}{E_{s}},
$$

where $E_{r}$ and $E_{s}$ are the average model energy of the interesting subsystem and the source subsystem, respectively, and $E_{r}^{i}$ represents the contributed energy of the $i$ th energy transfer path to the overall energy of the interesting subsystem.

Separating (6) out, the contribution of each energy transfer path, also named the path loss factor [12], is described as the following equation:

$$
D^{i}=\frac{E_{r}^{i}}{E_{s}}
$$

For example, there are $n$ subsystems taking part in the route of the $i$ th energy transfer path. From the source subsystem to the interesting subsystem, these acting subsystems are connected in the form of exchanging energy and supposed to be coupled one by one without the situation of one subsystem couples with two subsystems. Actually, one subsystem often couples with several other subsystems. If a subsystem couples with other two subsystems, the situation will be 


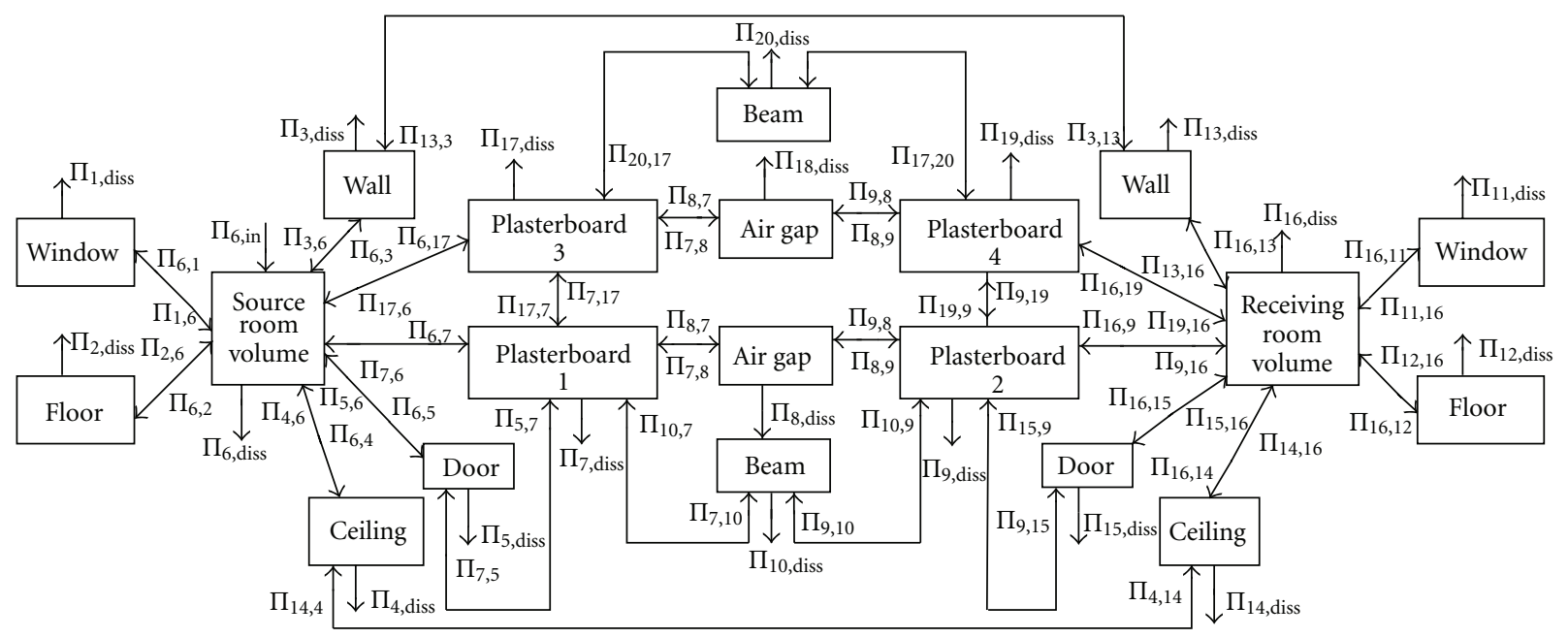

FIGURE 3: The energy flow relationships between subsystems after noise control.

treated as two different paths respectively. Taking the first energy transfer path as a example, assuming there are three subsystems in the middle of this transmission path, denoting these three subsystems as the $d$ th, eth, and $f$ th subsystems, then the path loss factor is computed as

$$
D^{1}=\frac{E_{r}^{1}}{E_{s}}=\frac{\eta_{s_{-} d} \cdot \eta_{d_{-} e} \cdot \eta_{e_{-} f} \cdot \eta_{f_{-} r}}{\eta_{d} \cdot \eta_{e} \cdot \eta_{f} \cdot \eta_{r}}
$$

where $\eta_{s_{-} d}$ is the CLF between the source subsystem and the $d$ th subsystem, and the physical meanings are analogically for $\eta_{d_{e} e}, \eta_{e_{-}}$, and $\eta_{f_{-} r}$. Symbols $\eta_{d}, \eta_{e}, \eta_{f}$, and $\eta_{r}$ are the DLFs of subsystem $d, e, f$ and the interesting subsystem, respectively.

Equation (7) is divided by (6), and the percentile of each contributor can be obtained

$$
P^{i}=\frac{D^{i}}{D}=\frac{D^{i}}{\sum_{i} D^{i}}
$$

\section{Experimental Studies}

In order to understand the acoustical properties of the pump and obtain data for calculation and validation, some experimental work was finished firstly before noise control. The motor of the pump runs in a rotor speed around $2900 \mathrm{r} / \mathrm{min}$, which is the normal used speed but not the maximal one. The measurement locations are distributed symmetrically in the center line of the source room and receiving room respectively. The measured average sound pressure levels are shown in Figure 4.

From the curves in Figure 4, it can be found that the maximum response in both rooms is around $1.25 \mathrm{kHz}$, which is the harmonic frequency of the rotor. This frequency is in the sensitive frequency range of human hearing, around $1 \mathrm{kHz}$. Consequently, a person in the receiving room will feel very uncomfortable if the pump works.

Furthermore, attention should be paid to the overall sound pressure level, which is $88.7 \mathrm{~dB}(\mathrm{~A})$ in the source room and $84.7 \mathrm{~dB}(\mathrm{~A})$ in the receiving room, respectively. Unfortunately, the sound pressure level in the source room exceeds

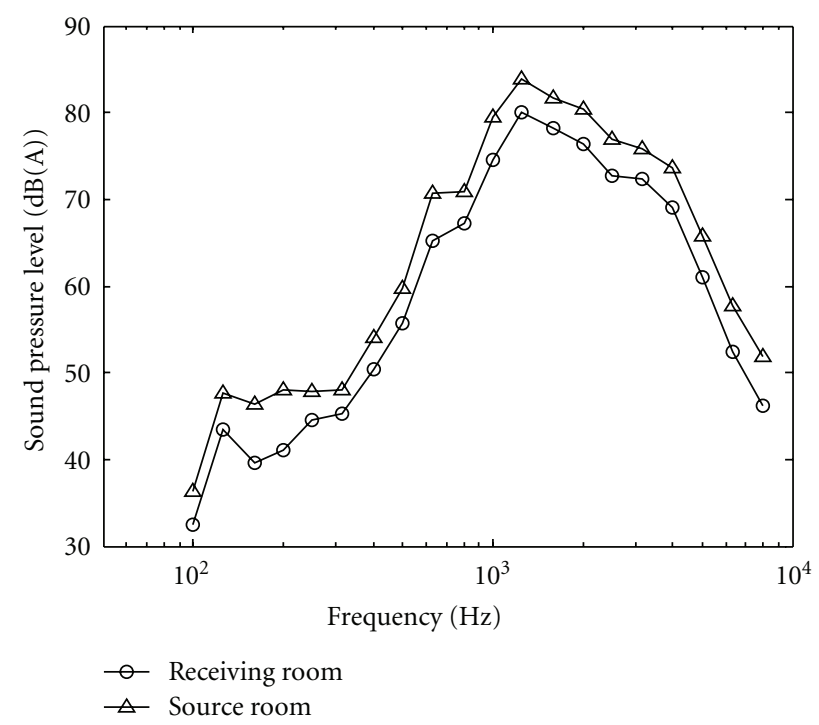

FIGURE 4: Measured sound pressure levels before noise control.

the regulation of $85 \mathrm{~dB}(\mathrm{~A})$. However, the value in the receiving room just arrives the limitation, but it will also exceed $85 \mathrm{~dB}(\mathrm{~A})$ if the pump runs in a speed which is a little larger than $2900 \mathrm{r} / \mathrm{m}$.

From the above analysis of measured results in coupled workshops, it is required that noise treatment should take place. The ascendant decision is to fill the opening. A single side of the double plasterboards used for filling the opening is shown in Figure 5. The thickness is $12.5 \mathrm{~mm}$.

Similarly, the measurement of acoustical responses was also carried out after noise treatment, and the sound pressure levels are plotted in Figure 6.

Damping loss factor (DLF) is one of the basic parameters in SEA modeling and used for predicting the power dissipated inside the subsystem. Because it is unrealistic to measure the DLF of structural subsystem separately, approximate values of concrete, brickwork, plywood, and 


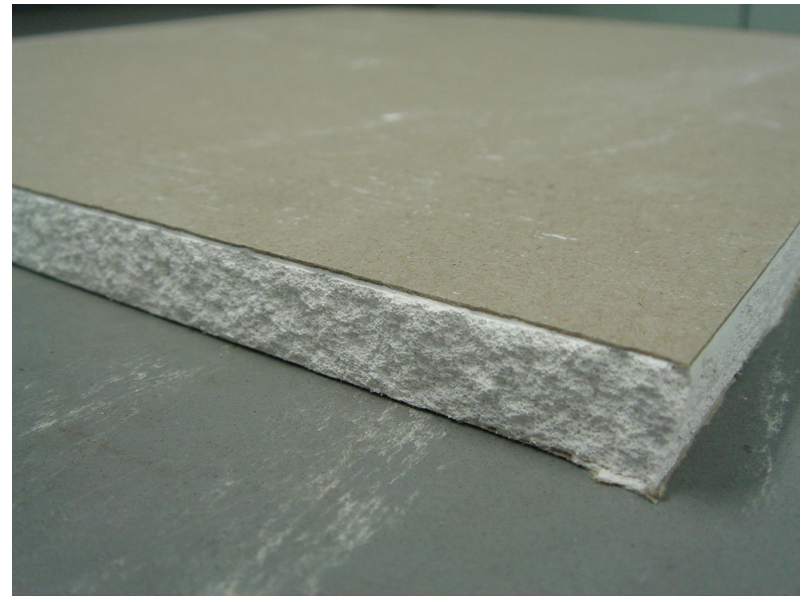

FIGURE 5: The plasterboard filling the opening used for noise control.

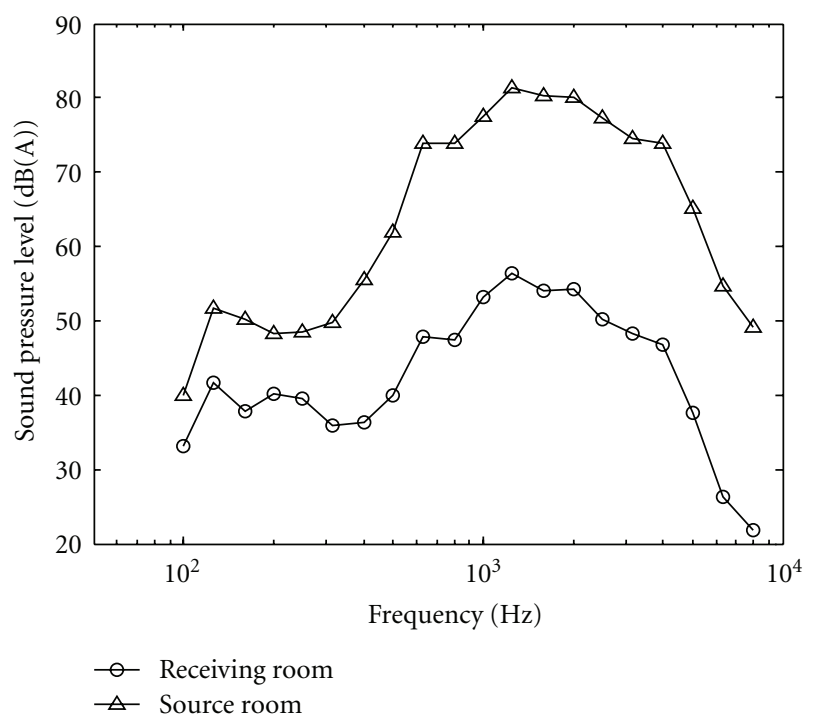

FIgURE 6: Measured sound pressure levels after noise control.

glass are cited from [13]. Jaime and Manuel [14] proposed a fitting formula for computing DLF of plasterboard, as follows:

$$
\eta_{d_{-} \text {plasterboard }}=0.4171 f^{-0.4467} .
$$

The DLF of room volumes can be calculated by measuring their reverberation time $T_{60}$. The relationship between the DLF and the reverberation time $T_{60}$ can be obtained from the half-power bandwidth method and is expressed as follows:

$$
\eta_{d}=\frac{2.2}{f T_{60}} .
$$

The measured DLFs of the room volumes before and after noise control are shown in Figure 7. Because the source power is sufficient above $100 \mathrm{~Hz}$, the measured results are reliable. It can be found that the DLFs of these two workshops have a great agreement above $400 \mathrm{~Hz}$ before control and have little difference at almost all frequencies after control.

\section{Results and Discussion}

5.1. Before Noise Control. The detailed values of material property and DLF are listed in Table 1.

Based on above analysis, using the acoustical response in the source room as the input condition for (1), the predicted sound pressure level difference between the coupled workshops is plotted in Figure 8. Error range of $3 \mathrm{~dB}$ is given in the figure for analyzing the deviation between the predicted and measured results. There are many causes for the prediction error. From [6], if the sound transmission coefficient through the opening equals unity, the maximum error is $2 \mathrm{~dB}$. The measurement error is setting to $1 \mathrm{~dB}$ in sound pressure level meter (B\&K2238) during experiment. Hence, the total prediction error is larger than $3 \mathrm{~dB}$ because of an other undetermined error coming from hypotheses proposed in the foregoing section.

From the results in Figure 8, it is found that the maximum noise reduction is nearly $7 \mathrm{~dB}(\mathrm{~A})$ around $200 \mathrm{~Hz}$ and the average noise reduction is $4 \mathrm{~dB}(\mathrm{~A})$ from $100 \mathrm{~Hz}$ to $8 \mathrm{kHz}$. It is noticeable that most of the measured results are in the error bar and the maximum deviation is approximately $5 \mathrm{~dB}(\mathrm{~A})$ at $160 \mathrm{~Hz}$ and $200 \mathrm{~Hz}$, so the theoretical model is reliable.

In addition, the contribution of the most important energy transfer path and other ones is plotted in Figure 9, where the quantity of $y$-axis is the contribution in the form of percentage. Figure 9(a) represents that the sound energy in the source room transmits through the opening to the receiving room, and Figure 9 (b) is the contribution of other paths except that in Figure 9(a). It is shown that the most important energy transfer way is that the sound energy transmits through the opening directly, and the percentage is $15 \%$ at a low frequency of around $100 \mathrm{~Hz}$ and increases smoothly until $400 \mathrm{~Hz}$ to more than $95 \%$, and the value has no evident changes except $3.15 \mathrm{kHz}$ with the percent of $83 \%$. The comparison between the most significant path and other ones indicated that the treatment should be focused on the opening and other contributors can be ignored, especially at the intermediate and high frequencies.

5.2. After Noise Control. From the prediction and analysis before noise control, it is affirmed that most of the sound energy in the noisy source room is transmitted to the receiving room through the opening and filling it is a straightforward way to control the noise level in the receiving room. But if leakage appears during the treatment work, the noise reduction will be affected remarkably [7]. Consequently, analyzing the noise reduction with a different leakage area is meaningful to practice. Figure 10 gives three cases of configuration of a different leakage area that will be discussed in the following content. The different area is described by different width and consistent length and depth. The length is $15 \mathrm{~m}$ and the depth is $0.09 \mathrm{~m}$. 


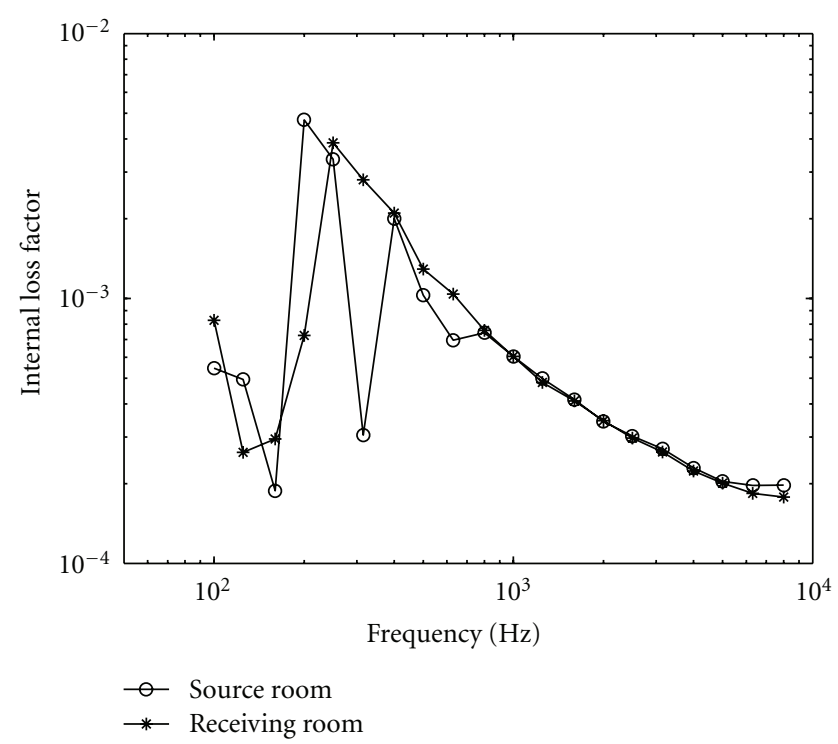

(a) Before noise control

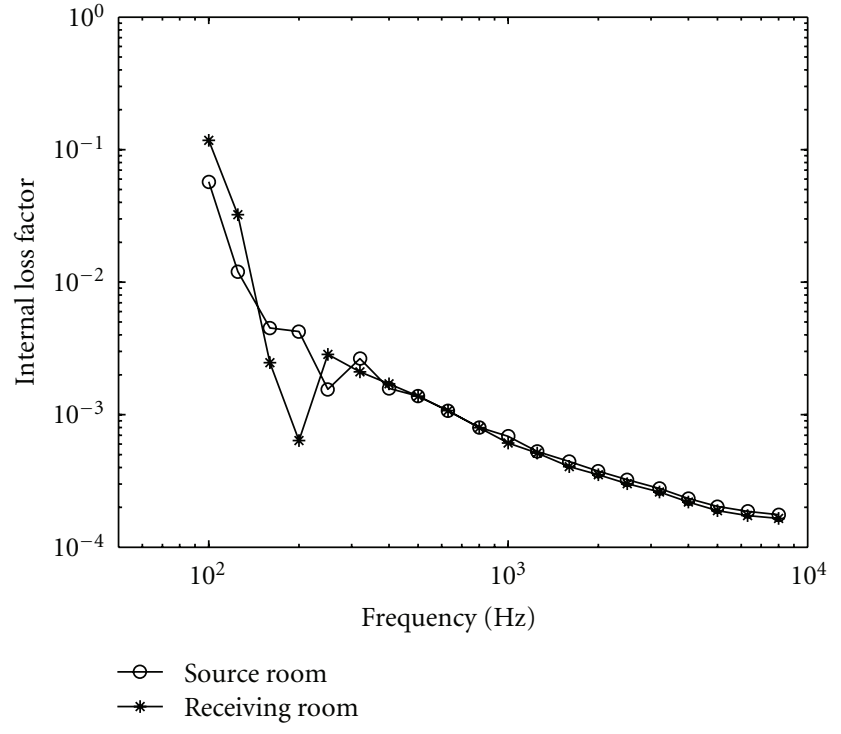

(b) After noise control

FIGURE 7: The DLFs of the coupled room volumes.

TABLE 1: The material properties and DLFs of structures.

\begin{tabular}{lcccc}
\hline Material & Density kg/m & Young's modulus Pa & Poisson's ratio & DLF \\
\hline Concrete (floors) & 1900 & $1.6 \times 10^{10}$ & 0.25 & 0.02 \\
Brickwork (walls) & 1900 & $1.6 \times 10^{10}$ & 0.2 & 0.02 \\
Plasterboard (clapboard and ceiling) & 800 & $2 \times 10^{9}$ & 0.26 & Calculated by Jaime et al. [14]'s formula \\
Plywood (doors) & 700 & $6 \times 10^{9}$ & 0.25 & 0.01 \\
Glass (windows) & 2300 & $6.2 \times 10^{10}$ & 0.24 & 0.01 \\
\hline
\end{tabular}

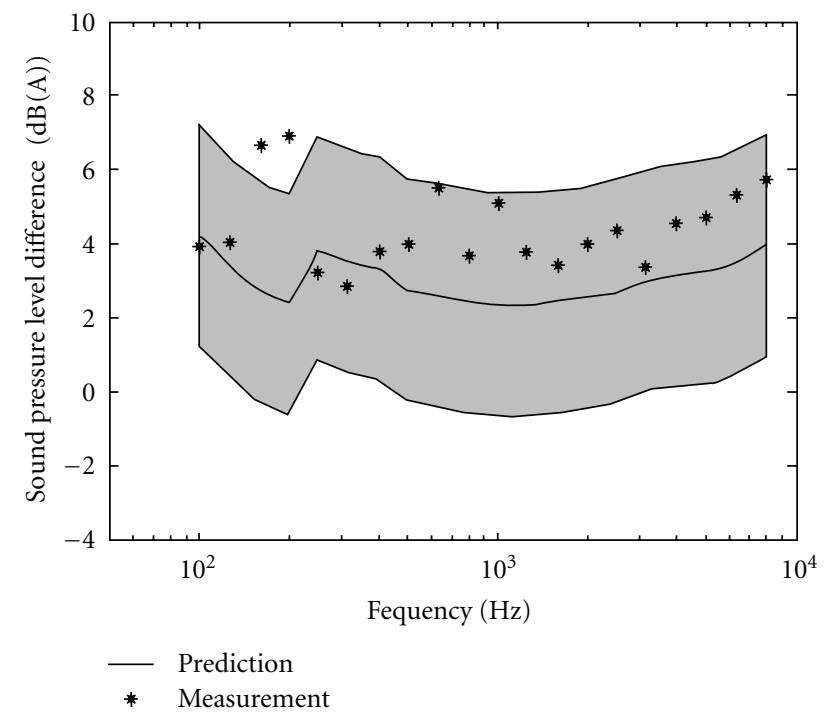

FIGURE 8: The sound pressure level difference before noise control.

The predicted sound pressure level differences, corresponding to the cases in Figure 10, are shown in Figure 11. It is shown apparently that the leakage is of great importance to the noise reduction. When there is no leakage existing between the designed and existing plasterboards, the effect of noise treatment is wonderful above $630 \mathrm{~Hz}$, and the average sound pressure level difference reaches $49 \mathrm{~dB}(\mathrm{~A})$ in the whole interesting frequency domain. If there is $0.1 \%$ leakage, the noise reduction will decline from $400 \mathrm{~Hz}$ of approximately $23 \mathrm{~dB}(\mathrm{~A})$ and reduce to approximately $20 \mathrm{~dB}(\mathrm{~A})$ at $8 \mathrm{kHz}$, compared with the values of no leakage, and the average sound pressure level difference is $20.8 \mathrm{~dB}(\mathrm{~A})$. When the area of leakage increases to $1 \%$ of the total area of the plasterboards, the noise reduction is about $5 \mathrm{~dB}(\mathrm{~A})$ higher than the quantity of no treatment at low frequencies and is almost the same with the value of $0.1 \%$ leakage at high frequencies; and the average sound pressure level difference drops to $13.2 \mathrm{~dB}(\mathrm{~A})$. It can be concluded that the area of leakage changing from zero to nonzero will affect the noise reduction at high frequencies more significantly and with the increase of the area of leakage, the influence of leak becomes more remarkable at low and intermediate frequencies.

According to the conclusion from Figure 11, it is suggested that the padding plasterboard should connect with the existing one as the more sealed, the better. Through observing the accomplished controlling work, there is no obvious leakage existing. Therefore, the SEA model with no leakage was used to predict the noise reduction after control. The measured and predicted sound pressure level difference is drawn in Figure 12. The highest analyzing frequency is 


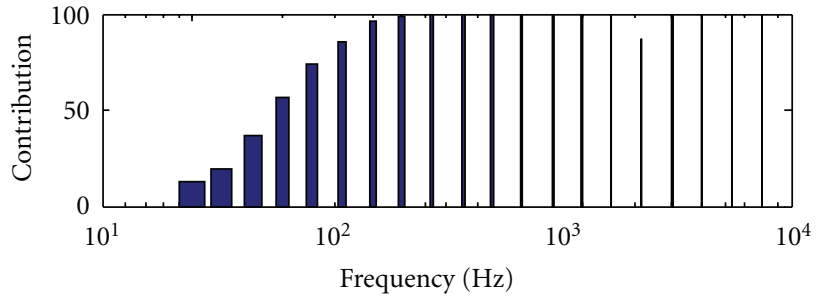

(a)

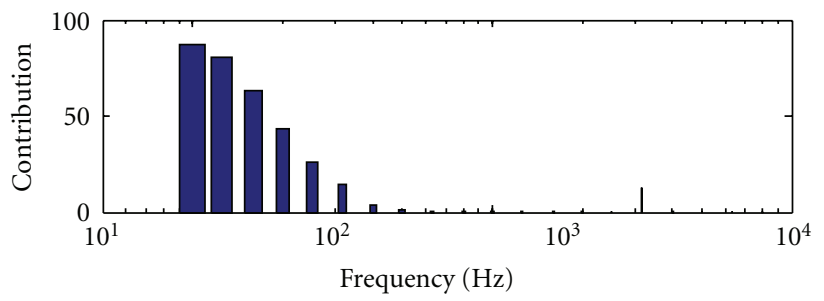

(b)

Figure 9: The contribution of energy transfer paths before noise control ((a): the energy transfer path from the source room through opening to the receiving room; (b): other energy transfer paths except subfigure (a)).

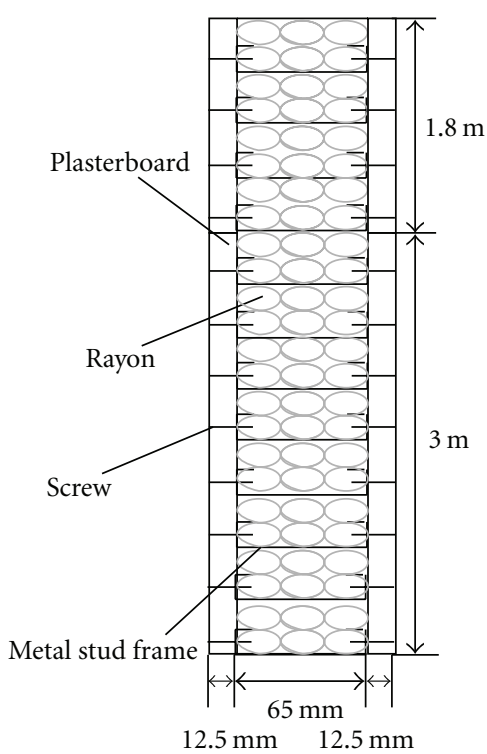

(a) Case 1

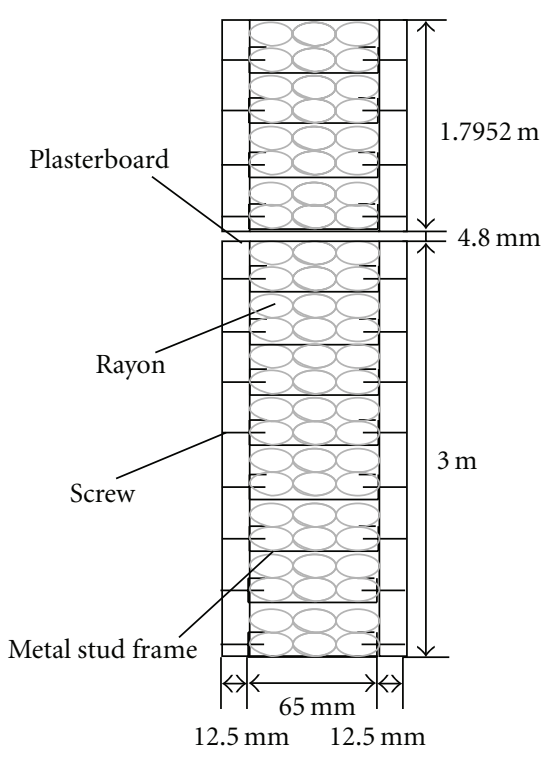

(b) Case 2

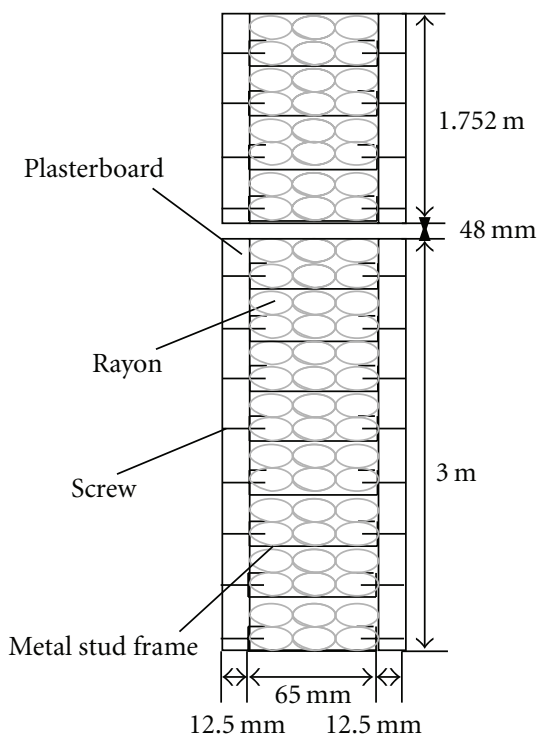

(c) Case 3

FIGURE 10: The plasterboard after noise control with a different area of leakage (Case 1: no leakage and the padding plasterboard connects with the existing one very well; Case 2: the area of the leakage occupies $0.1 \%$ of the total area of the plasterboards; Case 3: the area of the leakage occupies $1 \%$ of the total area of the plasterboards).

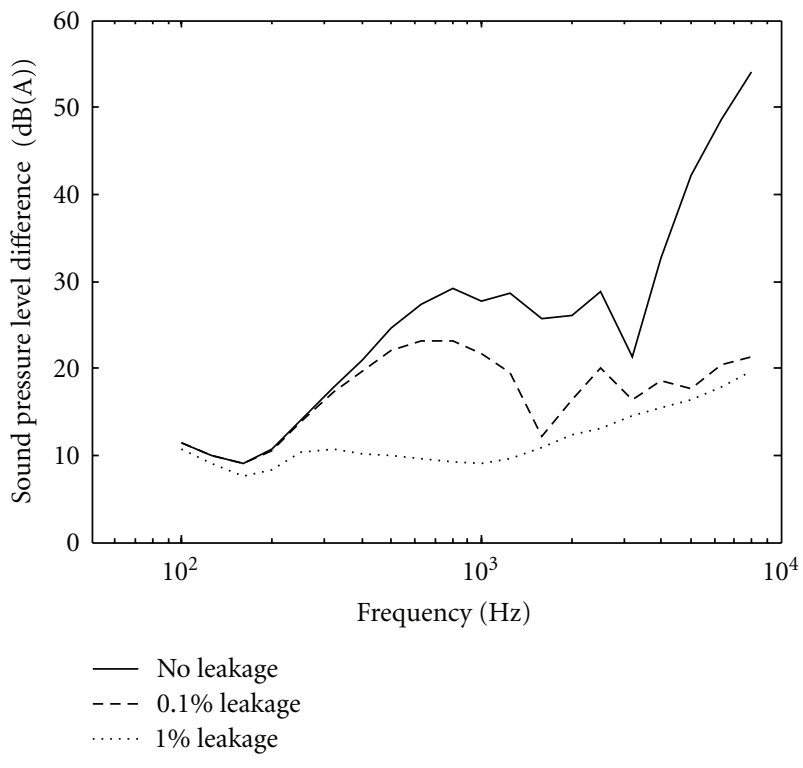

FIGURE 11: The sound pressure level difference after noise control.
$4 \mathrm{kHz}$, because of the insufficient signal noise ratio, which is different from the results before control.

The average noise reduction is $26.7 \mathrm{~dB}(\mathrm{~A})$ and the maximum reduction of noise is about $30 \mathrm{~dB}(\mathrm{~A})$. Almost all measured data drops in the error range of $3 \mathrm{~dB}$, so the reliability of theoretical model is validated again.

\section{Conclusions}

Some research on predicting noise reduction and controlling noise in coupled workshops was finished by using SEA method, including the cases of considering opening, leakage, and perfect situation without sound leak. The opening was taken as a filter in which the transmission coefficient between the coupled workshops through it equals unity. The leakage was taken into consideration by adopting the model of Gomperts et al..

The measured sound pressure level before noise control affirmed that the acoustical responses in both workshops exceed the noise exposure regulation of workplace and need 


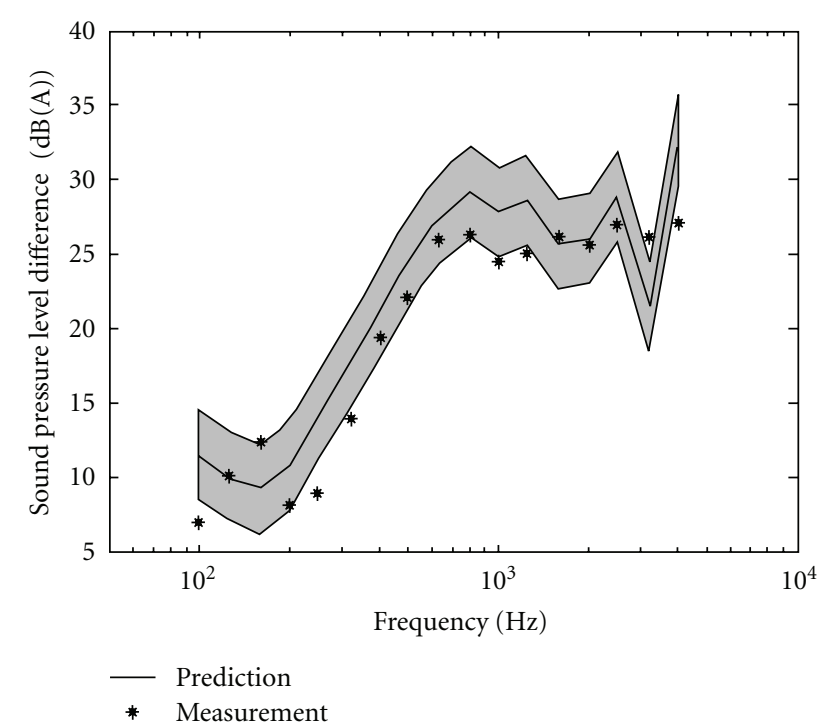

FIGURE 12: The sound pressure level difference after noise control.

to be controlled. Before treatment, most of the measured sound pressure level difference is in the error bar of prediction of $3 \mathrm{~dB}$. The average noise reduction is $4 \mathrm{~dB}(\mathrm{~A})$ and the most important way to transfer sound energy is through the opening directly and occupies more than $95 \%$ of the total contributors above $400 \mathrm{~Hz}$.

After noise control, the measured results also have good agreement with the prediction. By comparing the noise reduction before and after control, the sound pressure level difference increases by $22.7 \mathrm{~dB}(\mathrm{~A})$ in the form of overall sound pressure level. It is also concluded that leakage is significant to noise reduction. When the leakage changes from none to existing, the effect of decreasing noise reduction is more observable at high frequencies, and with the increment of the leakage area, the noise reduction at low and intermediate frequencies is affected more remarkably.

\section{Acknowledgments}

This research has been undertaken in the University of Western Australia (UWA), under the joined PhD program between the Northwestern Polytechnical Universtiy (NWPU). Meanwhile, the experiment work is finished in the School of Civil and Mechanical Engineering in UWA and has received support from Mr. Rob Greenhalgh for organizing and arrangement. The group of Professor Krish Thiagarajan, Dr. Hongmei Shun, Dr. Yanni Zhang, and Dr. Ye Zhihui also provided assistance for the measurement work. The financial supports from UWA and the joined program and all the above-mentioned people are gratefully acknowledged.

\section{References}

[1] "National Code of Practice for Occupational Noise NOHSC: 2009," Published by the National Occupational Health and Safety Commission, 2004.
[2] H. Furukawa, K. Fujiwara, Y. Ando, and Z. I. Maekawa, "Analysis of the structure-borne sound in an existing building by the SEA method," Applied Acoustics, vol. 29, no. 4, pp. 255$271,1990$.

[3] R. Wentang and K. Attenborough, "Prediction of sound fields in rooms using statistical energy analysis," Applied Acoustics, vol. 34, no. 3, pp. 207-220, 1991.

[4] A. Sauter Jr. and W. W. Soroka, "Sound transmission through rectangular slots of finite depth between reverberant rooms," Journal of the Acoustic Society of America, vol. 47, no. 1A, pp. 5-11, 1969.

[5] A. Billon, V. Valeau, A. Sakout, and J. Picaut, "On the use of a diffusion model for acoustically coupled rooms," Journal of the Acoustical Society of America, vol. 120, no. 4, pp. 2043-2054, 2006.

[6] S. Franck, N. Hugues, and A. Nouredddine, "On the modeling of the diffuse field sound transmission loss of finite thickness apertures," Journal of the Acoustical Society of America, vol. 122, no. 1, pp. 302-313, 2007.

[7] M. C. Gomperts and T. Kihlman, "The sound transmission loss of circular and slit-shaped aperture in walls," Acustica, vol. 18, pp. 144-150, 1967.

[8] Y. Lei, J. Pan, and M. P. Sheng, "Noise reduction of an acoustical enclosure-mechanisms and prediction accuracy," in Proceedings of 20th International Congress on Acoustics, Sydney, Australia, August 2010.

[9] R. H. Lyon, Statistical Energy Analysis of Dynamical Systems: Theory and Applications, M.I.T. Press, Cambridge, Mass, USA, 1975.

[10] T. R. T. Nightingale and I. Bosmans, "Expressions for firstorder flanking paths in homogeneous isotropic and lightly damped buildings," Acta Acustica, vol. 89, no. 1, pp. 110-122, 2003.

[11] I. L. Ver and C. I. Holmer, "Interaction of sound waves with solid structures," in Noise and Vibration Control, L. L. Beranek, Ed., McGraw-Hill, New York, NY, USA, 1971.

[12] A. T. Chavan and D. N. Manik, "Design sensitivity analysis of statistical energy analysis models using transfer path approach," Electronic Journal Technical Acoustics, vol. 3, 2005.

[13] M. Dayou, Handbook of Noise and Vibration Controlling Engineering, China Machine Press, Beijing, China, 2002.

[14] D. Jaime and R. Manuel, "Loss factor measurements on plasterboard," in Proceedings of 19th International Congress on Acoustics, Madrid, Spain, September 2007. 

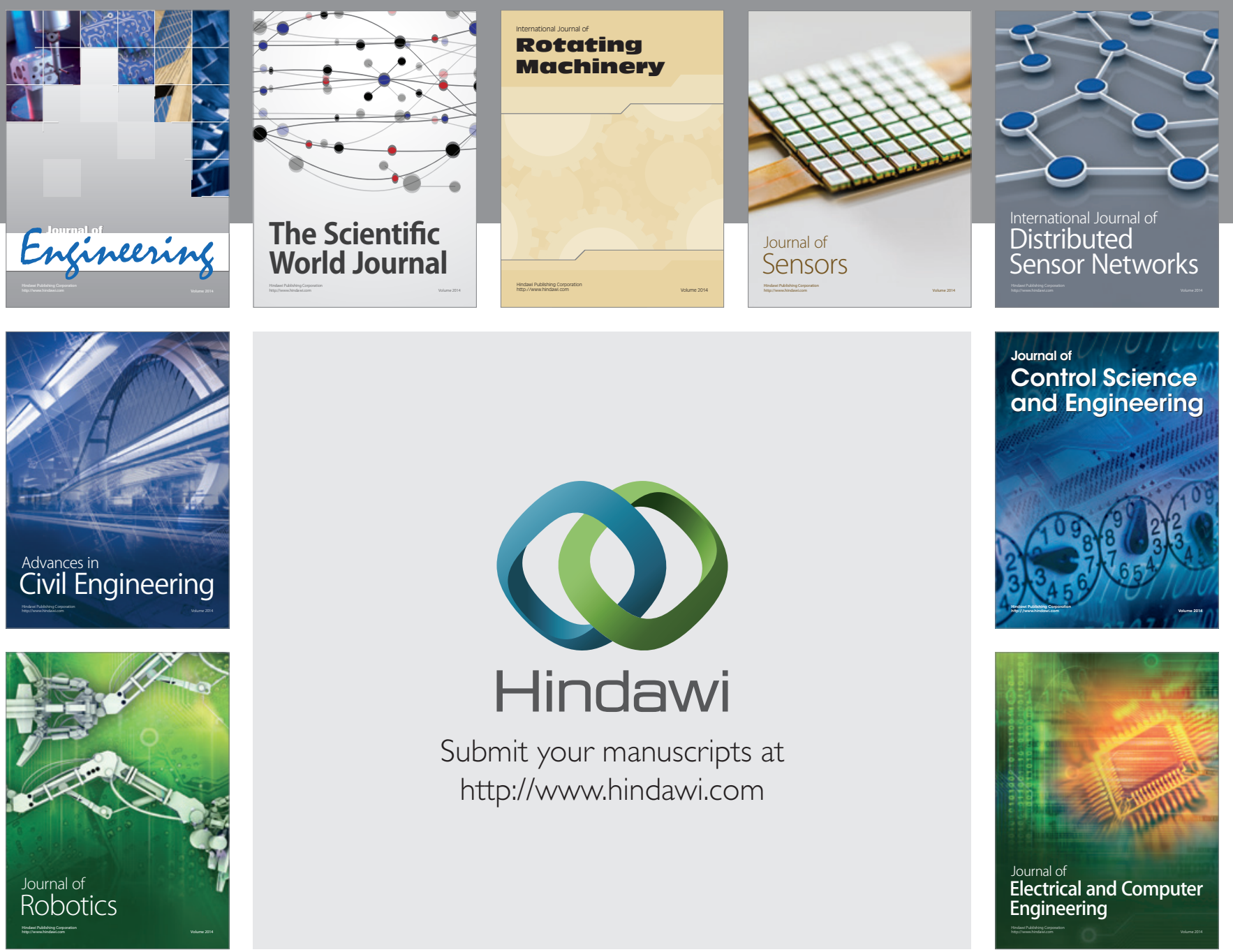

Submit your manuscripts at

http://www.hindawi.com
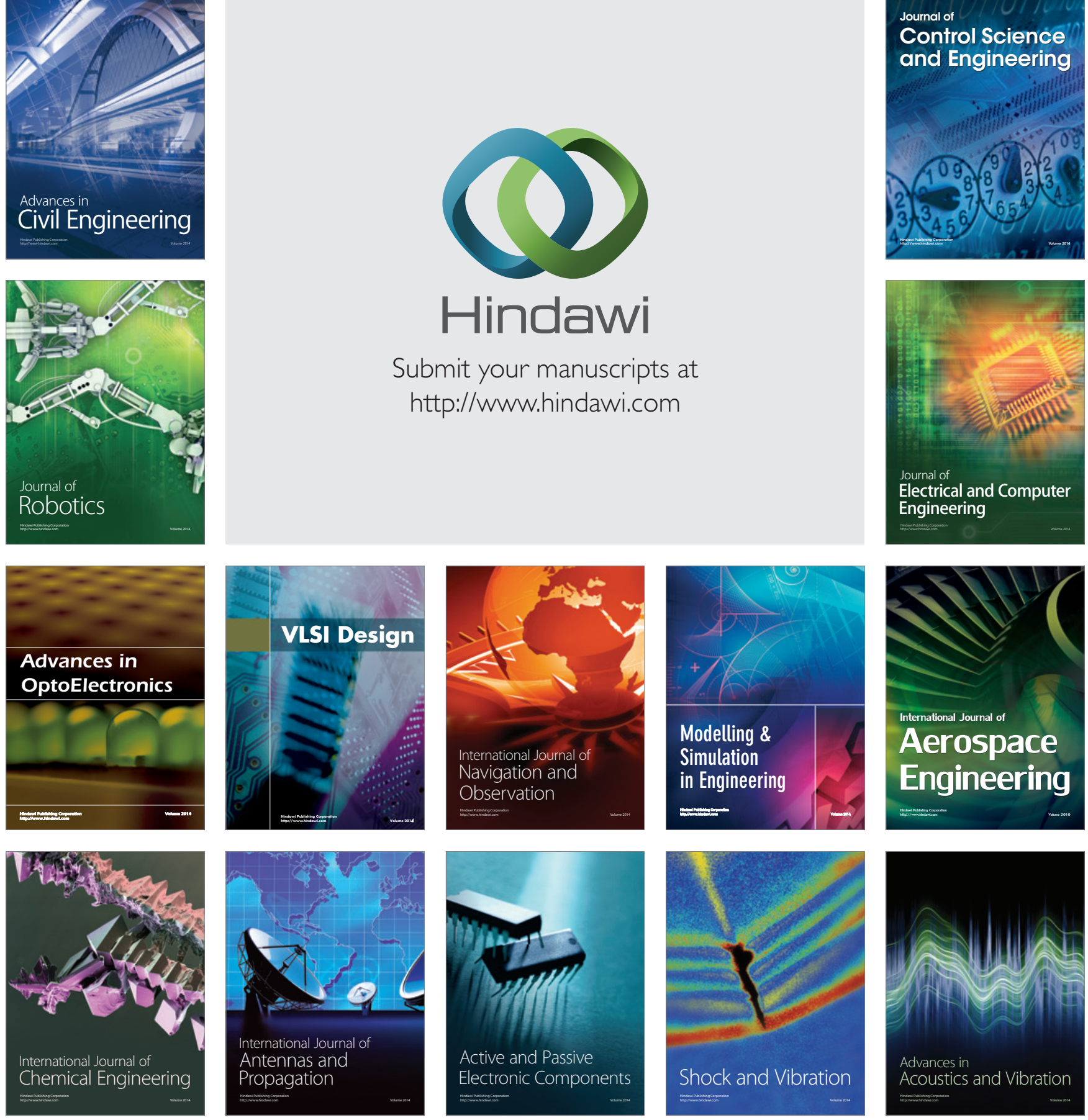